\title{
Propuesta de actualización de la evaluación antropométrica del recién nacido
}

\author{
Proposal to update the anthropometric evaluation of the newborn
}

\author{
Comité Nacional de Crecimiento y Desarrollo y Comité de Estudios Fetoneonatales (CEFEN)
}

\section{RESUMEN}

Desde el año 1986, se utilizan las referencias de crecimiento depeso, longitud corporal y perímetro cefálico para la evaluación antropométrica del recién nacido publicadas por $\mathrm{H}$. Lejarraga y $\mathrm{C}$. Fustiñana. Estas curvas fueron construidas con datos locales y extranjeros y se contó con escasos datos a edades neonatales tempranas. En ese momento,lasupervivencia delosniños prematuros extremos era muy baja y no se disponía de la infraestructura y los tratamientos actuales quehan permitidolasupervivenciadeniños prematuros de hasta 24-25 semanas de edad gestacional. Por ello, es necesario actualizar estas referencias.

Fenton y Kim, en el año 2013, revisaron los gráficos de crecimiento de prematuros del año 2003 e incluyeron datos recientes. Suavizaron los datos de prematurose incorporaron los estimados por la Organización Mundial de la Salud para ambos sexos.

La comparación de las curvas de Lejarraga y Fustiñana con las de Fenton muestra que el peso en promedio es menor en las curvas de Fenton. Con respecto a la longitud corporal y a partir de la semana 34 de edad gestacional, los niños de las curvas de Fenton tienen menor longitud corporal y perímetro cefálico en relación con los datos de Lejarraga y Fustiñana.

Las curvas de Fenton y Kim están disponibles entre las 22 y las 50 semanas posconcepcionales para ambos sexos y empalman en la semana 50 posconcepcional con las curvas para niños de término en su semana 10 posnatal de la Organización Mundial de la Salud, curvas adoptadas por laSociedad Argentina de Pediatría y el Ministerio de Salud.

Sobre estas bases, proponemos y avalamos el reemplazodelascurvasdeLejarragay Fustiñanapor las de Fenton y Kim para el seguimiento del recién nacido prematuro durante la internación y posalta. Palabras clave: curvas de crecimiento intrauterino, recién nacido, peso al nacer, talla, antropometría.

http:/ /dx.doi.org/10.5546/aap.2017.89
Por mucho tiempo, se consideró el peso de nacimiento como una variable dicotómica: bajo peso y prematurez eran equivalentes para aquellos niños con peso inferior a 2500 gramos; el resto eran considerados niños de término. Pero, entre 1950 y 1960, hubo un gran aumento de información que diferenció entre bajo peso y prematurez. ${ }^{1}$ Esta concepción permitió que, desde hace poco más de 50 años, Lula Lubchenco propusiera el uso de curvas de peso al nacer en función de la edad gestacional (EG) como criterio de clasificación antropométrica del recién nacido $(\mathrm{RN}) .^{2}$ De manera similar, la primera publicación con datos argentinos fue la realizada por Lejarraga y col. en 1975 con recopilación de datos en el Hospital Materno Infantil Ramón Sardá. ${ }^{3}$

Desde el año 1986, y a partir de los datos del estudio anterior, junto con otros, se utilizan las referencias de crecimiento de peso, longitud corporal (LC) y perímetro cefálico (PC) para la evaluación antropométrica del RN publicadas por H. Lejarraga y C. Fustiñana. ${ }^{4}$ Estas curvas han tenido una amplia aceptación y uso en todo el país por pediatras $\mathrm{y}$, particularmente, neonatólogos, y fueron recomendadas y difundidas por el Ministerio de Salud de la Nación ${ }^{5}$ y la Sociedad Argentina de Pediatría. ${ }^{6}$
Correspondencia:

Dra. Mariana del Pino, mdelpino@intramed.net

Financiamiento:

Ninguno.

\section{Conflicto de intereses:} Ninguno que declarar.

Recibido: 16-6-2016 Aceptado: 11-8-2016
Comité Nacional de Crecimiento y Desarrollo: Dra. Liliana Villafañe, Dra. Mariana del Pino, Dra. Rocío Rabosto Moleón y Dr. Enrique Abeyá Gilardon. Comité de Estudios Fetoneonatales (CEFEN): Dra. Silvia Fernández Jonusas y Dr. Ricardo Nieto. 
Fueron construidas con datos de más de 40 años provenientes de una sola unidad neonatal sumados a datos extranjeros, algunos de los cuales datan del año sesenta. Dentro de sus problemas metodológicos, la toma de los datos antropométricos de las diferentes muestras fue heterogénea, con escasos datos a edades neonatales tempranas, por lo que se debió agrupar ambos sexos para calcular los sDE. Además, el cálculo de la EG se basó únicamente en la fecha de la última menstruación (FUM), lo que puede llevar a un error de estimación, sobre todo a EG extremas (prematurez y postérmino). ${ }^{7}$ Asimismo, los criterios auxológicos para la definición y la construcción de curvas de referencia han evolucionado a nuevos criterios. ${ }^{8}$

Cuando se elaboraron esas curvas, la supervivencia de los niños prematuros extremos (nacidos antes de la semana 28) era muy baja y no se disponía de la infraestructura y los tratamientos del presente. "Actualmente, la disponibilidad de nuevas terapéuticas, la creciente complejidad de las unidades de cuidados intensivos, la especialización en Neonatología y aún las subespecialidades de la misma han permitido la supervivencia de niños nacidos a las 24 o 25 semanas con algo más de 500 gramos al nacimiento". 9

La evaluación antropométrica del RN y su posterior seguimiento requieren de la medición del peso, la LC y el PC. Cada una de estas mediciones expresa aspectos diferentes del crecimiento y, por lo tanto, son complementarias entre sí, si bien la medición más usada es el peso. ${ }^{10}$ Para comparar las tres, es conveniente contar con las gráficas de las tres curvas en una misma hoja, pues facilita la interpretación.

La construcción de curvas con datos de RN prematuros es controvertida, ya que su crecimiento posnatal puede diferir del crecimiento intrauterino. ${ }^{11-15}$ Hay que tener en cuenta que la propia prematurez puede ser desencadenada por un proceso que esté interfiriendo en el crecimiento fetal, por lo que el RN prematuro podría ser más pequeño que un feto de la misma EG que esté intraútero. La utilización de referencias obtenidas con estos datos podría subestimar la incidencia real de $R N$ pequeños para la $E G$ con sus repercusiones en la clínica. Más allá de las debilidades que tienen las referencias construidas con datos de RN prematuros, estas están ampliamente aceptadas y utilizadas para realizar la evaluación auxológica del RN.

Las técnicas ecográficas de los últimos 25 años han permitido medir en forma longitudinal y estimar el peso fetal. Estos estudios han encontrado que el peso real del RN prematuro es menor que el estimado por ecografías. ${ }^{16,17}$ Además, la técnica ecográfica también tiene su error y cierto grado de imprecisión, y el cálculo del peso fetal estimado no es exacto, ya que se reporta una variabilidad de $+/-15 \%$ con respecto al peso real de nacimiento. Sin embargo, debe tenerse presente que las curvas ecográficas son útiles para el seguimiento y la evaluación del crecimiento intrauterino, pero no son idóneas para evaluar el peso al nacer. ${ }^{18}$

Aunque hay trabajos locales más recientes de peso al nacer según EG (San Pedro y col., ${ }^{19}$ Urquía y col. ${ }^{20}$ ), estos no incluyen datos de las otras mediciones de LC y PC. Además, en el primero, se incluyen solo datos de la mayor maternidad pública de la ciudad de Buenos Aires, donde se asiste el $20 \%$ de los nacimientos públicos de la ciudad, y, en el otro, la información proviene del dato primario del Informe Estadístico del Nacido Vivo de la Dirección de Estadísticas e Información en Salud (http:/ / www.deis.msal.gov.ar). Estos últimos datos son mediciones al nacer en forma no estandarizada con el error que conlleva.

Debido a lo expuesto anteriormente, es necesario actualizar las referencias de crecimiento para uso en el período neonatal, para que se cuente con datos sobre LC y PC, además del peso, y que se incluya a los prematuros extremos.

Dentro de las referencias utilizadas a nivel internacional, se encuentran las siguientes:

Fenton y Kim, ${ }^{21}$ en el año 2013, realizaron una revisión de los gráficos de crecimiento de prematuros publicados en el año 2003 con el objetivo de incluir datos recientes de tamaño al nacer, suavizar los datos de prematuros y los datos estimados por la Organización Mundial de la Salud (OMS), manteniendo los datos entre las 22 y las 36 semanas y a las 50 semanas de EG para ambos sexos.

Se incluyeron estudios poblacionales de crecimiento de pretérminos realizados en países desarrollados que contaron con percentilos a las semanas 24 o menores, de una muestra que incluyera, por lo menos, 500 niños menores de 30 semanas, recolectados en los últimos 25 años (1987-2012), teniendo en cuenta cualquier tendencia secular que pudiera existir.

Villar y col., ${ }^{22}$ publicaron en 2014, curvas de crecimiento de peso, LC y PC de RN de una muestra seleccionada bajo estrictos criterios, para tener una población con bajo riesgo de retardo de crecimiento fetal. Los gráficos presentan datos 
Figura 1. Comparación de los percentilos 3, 50 y 97 de las curvas de peso para niñas de Fenton y Kim (semanas 22-40) con las de Lejarraga y Fustiñana (semanas 27-40)

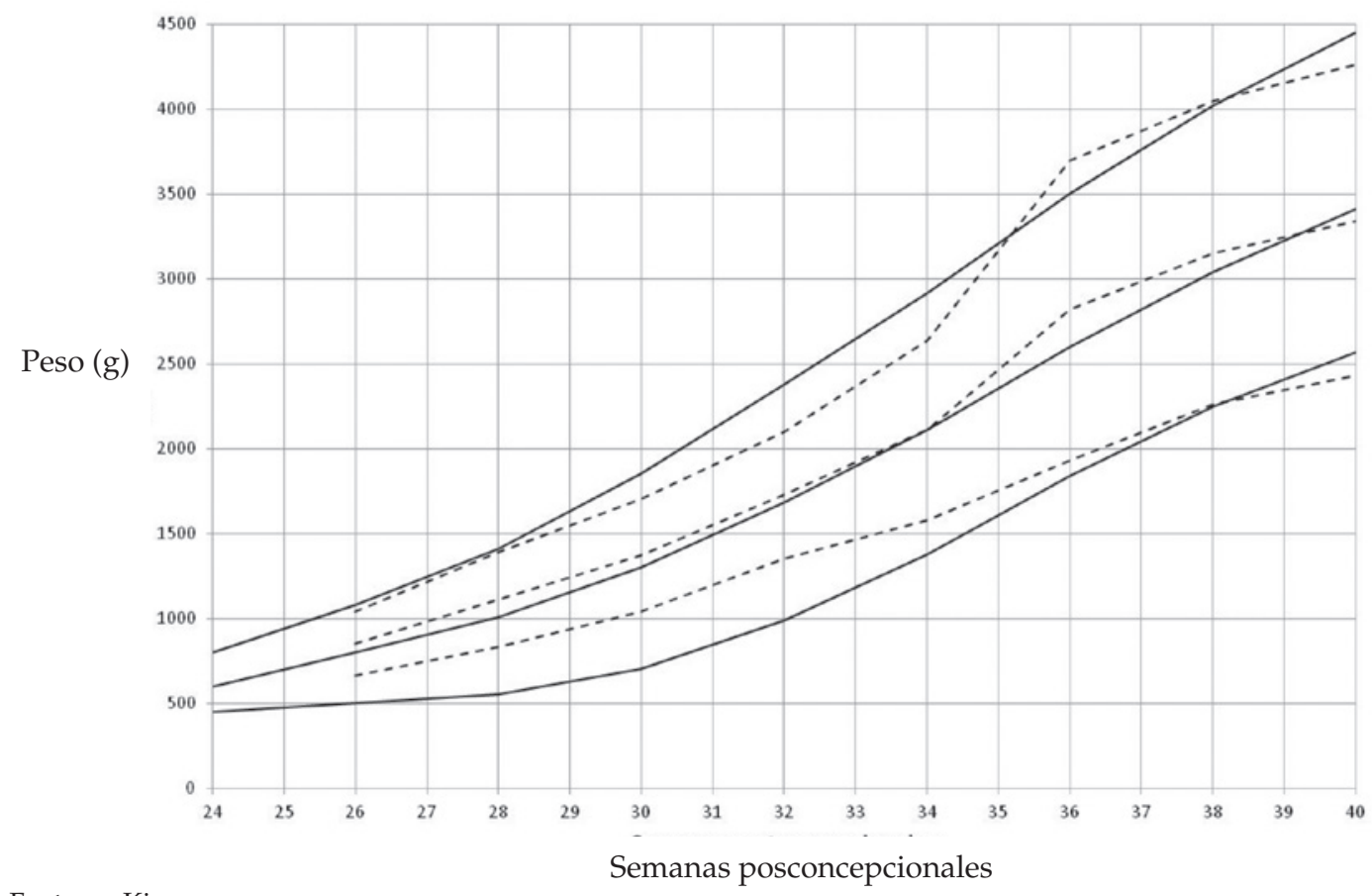

Fenton y Kim.

Lejarraga y Fustiñana.

Figura 2. Comparación de los percentilos 3, 50 y 97 de las curvas de peso para niños de Fenton y Kim (semanas 22-40) con las de Lejarraga y Fustiñana (semanas 27-40)

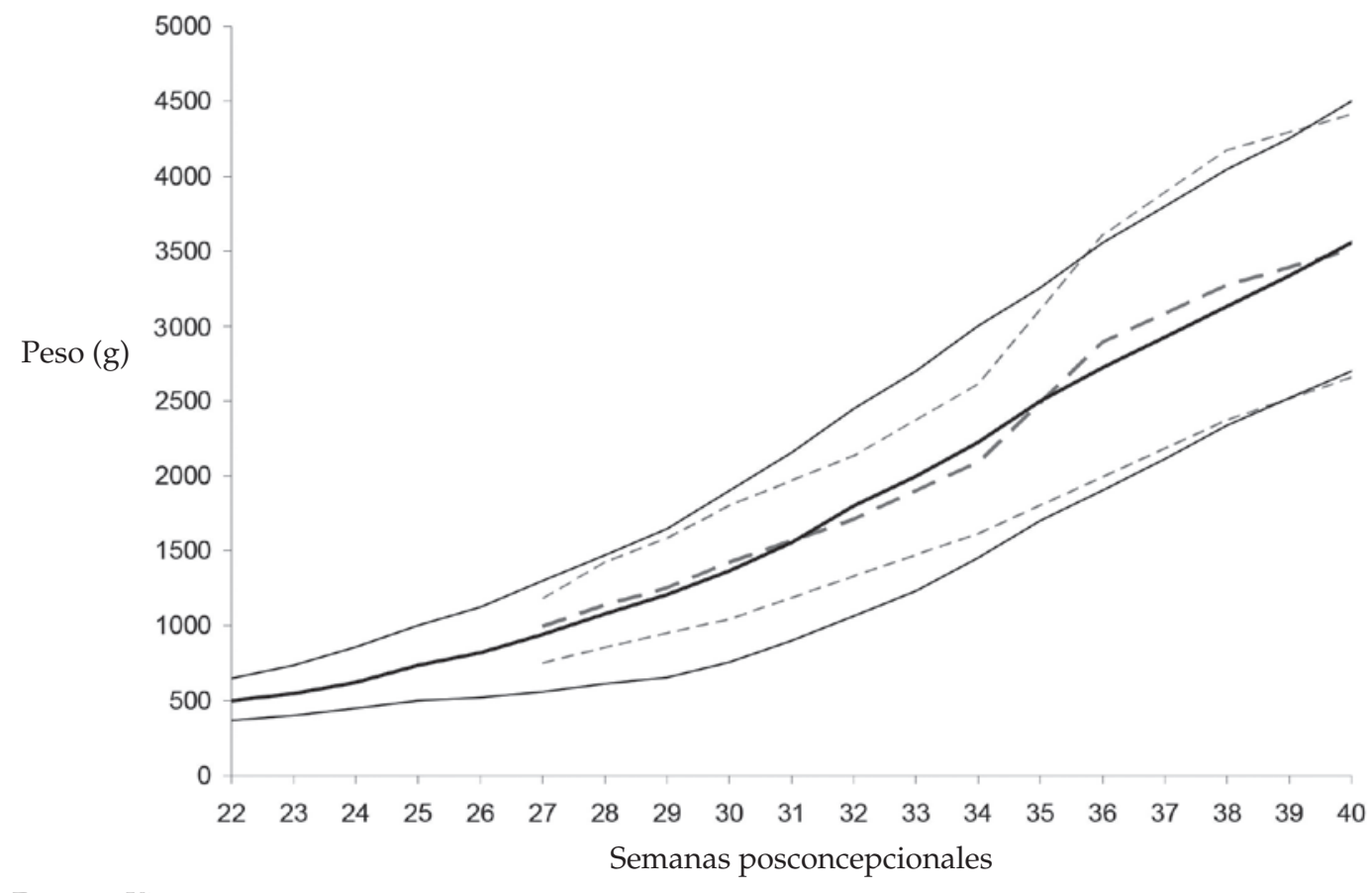

Fenton y Kim.

Lejarraga y Fustiñana. 
FIgURA 3. Comparación de los percentilos 3, 50 y 97 de las curvas de longitud corporal para niñas de Fenton y Kim (semanas 24-40) con las de Lejarraga y Fustiñana (semanas 26-40)

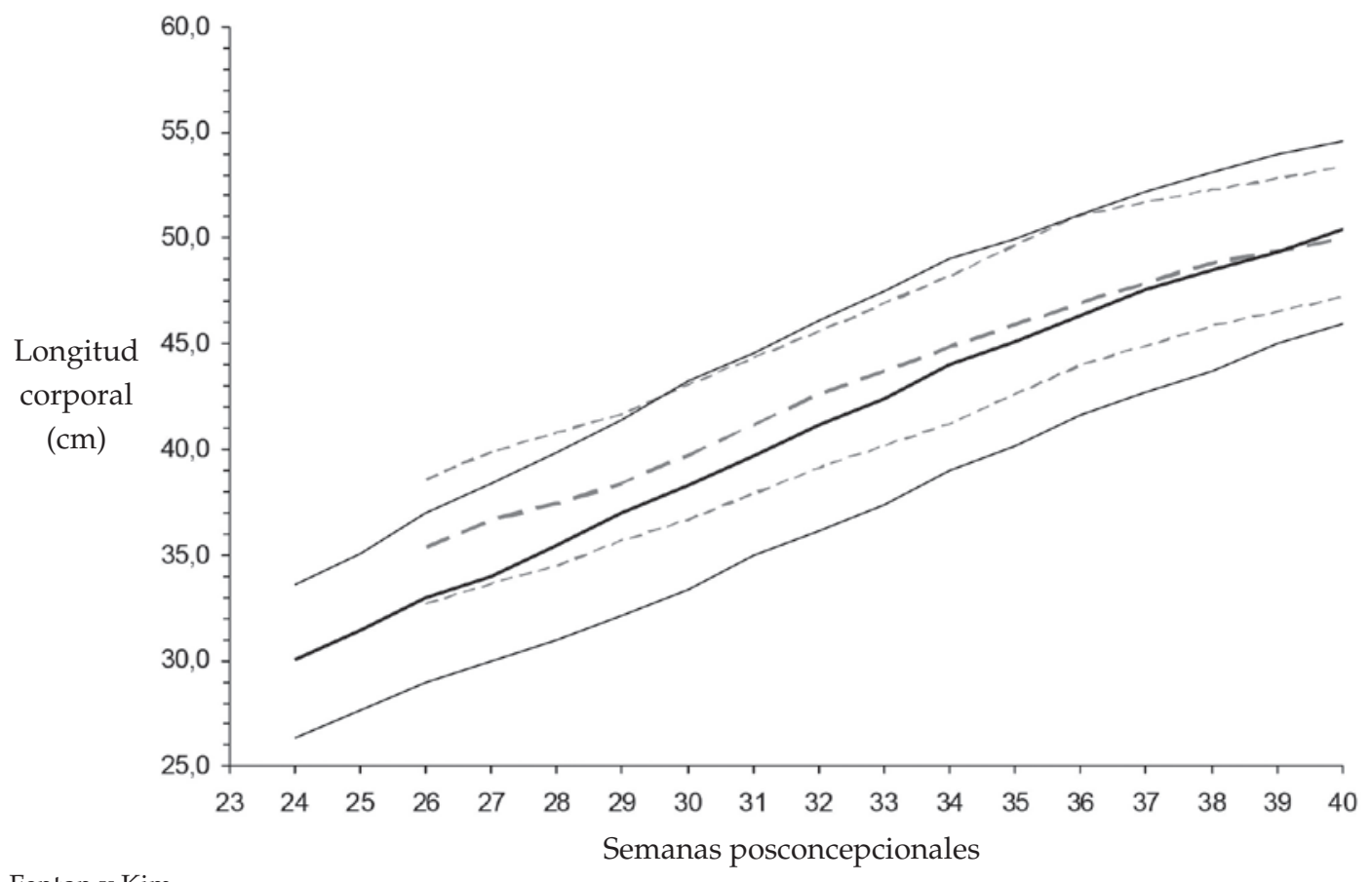

Fenton y Kim.

- - - - Lejarraga y Fustiñana.

FIgURA 4. Comparación de los percentilos 3, 50 y 97 de las curvas de longitud corporal para niños de Fenton y Kim (semanas 24-40) con las de Lejarraga y Fustiñana (semanas 26-40)

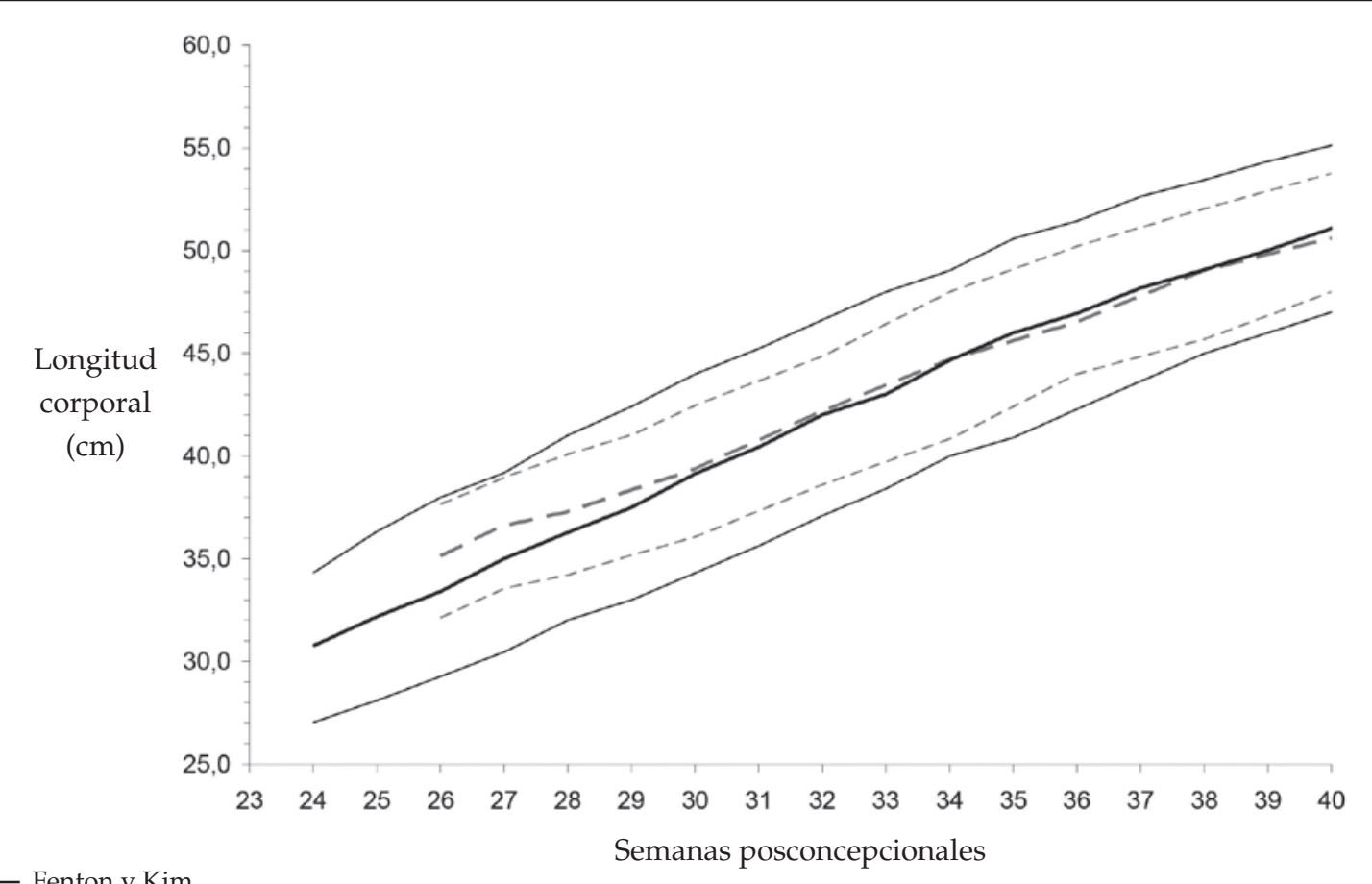

Fenton y Kim.

- - - Lejarraga y Fustiñana. 
Figura 5. Comparación de los percentilos 3, 50 y 97 de las curvas de perímetro cefálico para niñas de Fenton y Kim (semanas 24 a 40) con las de Lejarraga y Fustiñana (semanas 26 a 40)

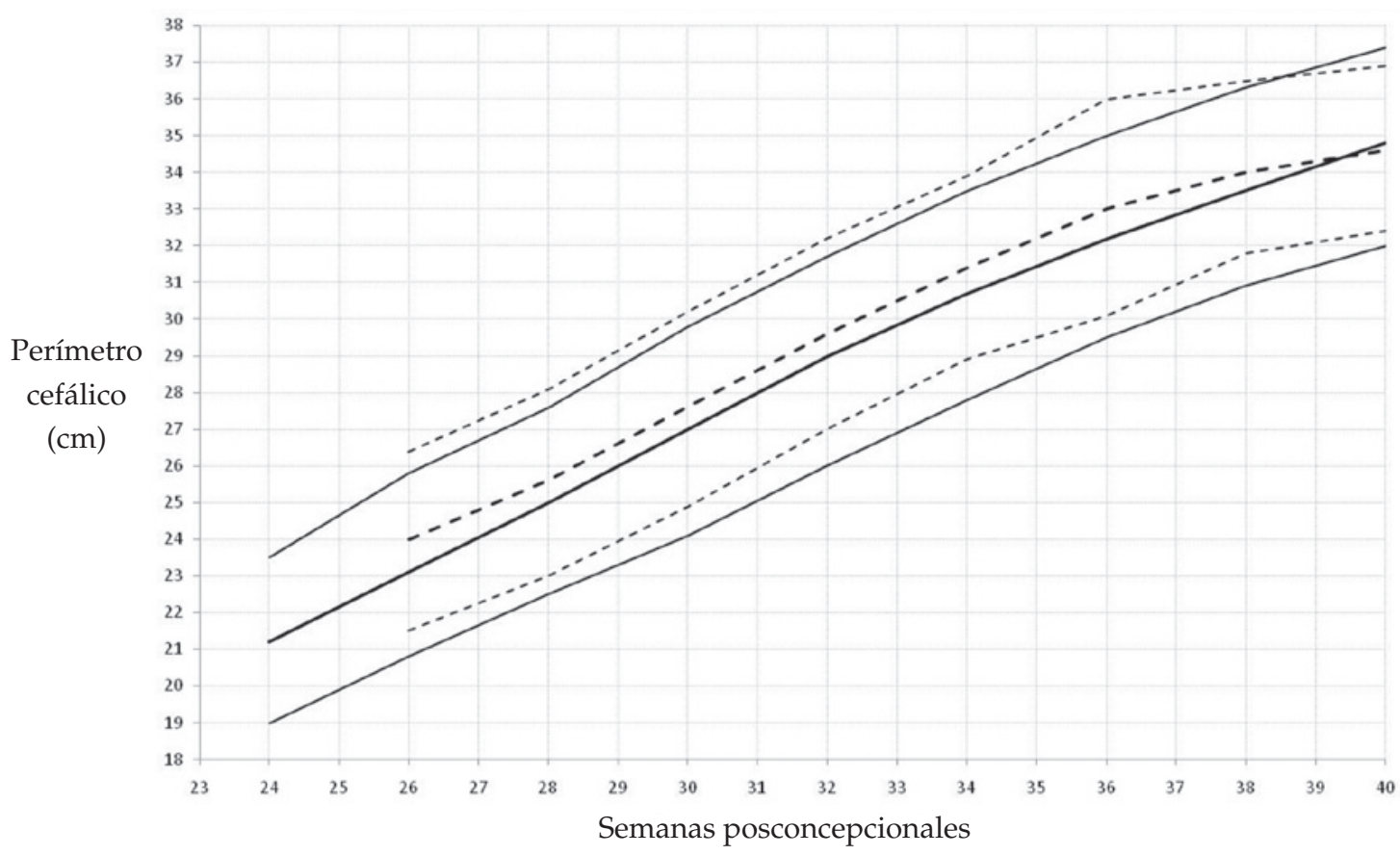

Figura 6. Comparación de los percentilos 3, 50 y 97 de las curvas de perímetro cefálico para niños de Fenton y Kim (semanas 24 a 40) con las de Lejarraga y Fustiñana (semanas 26 a 40)

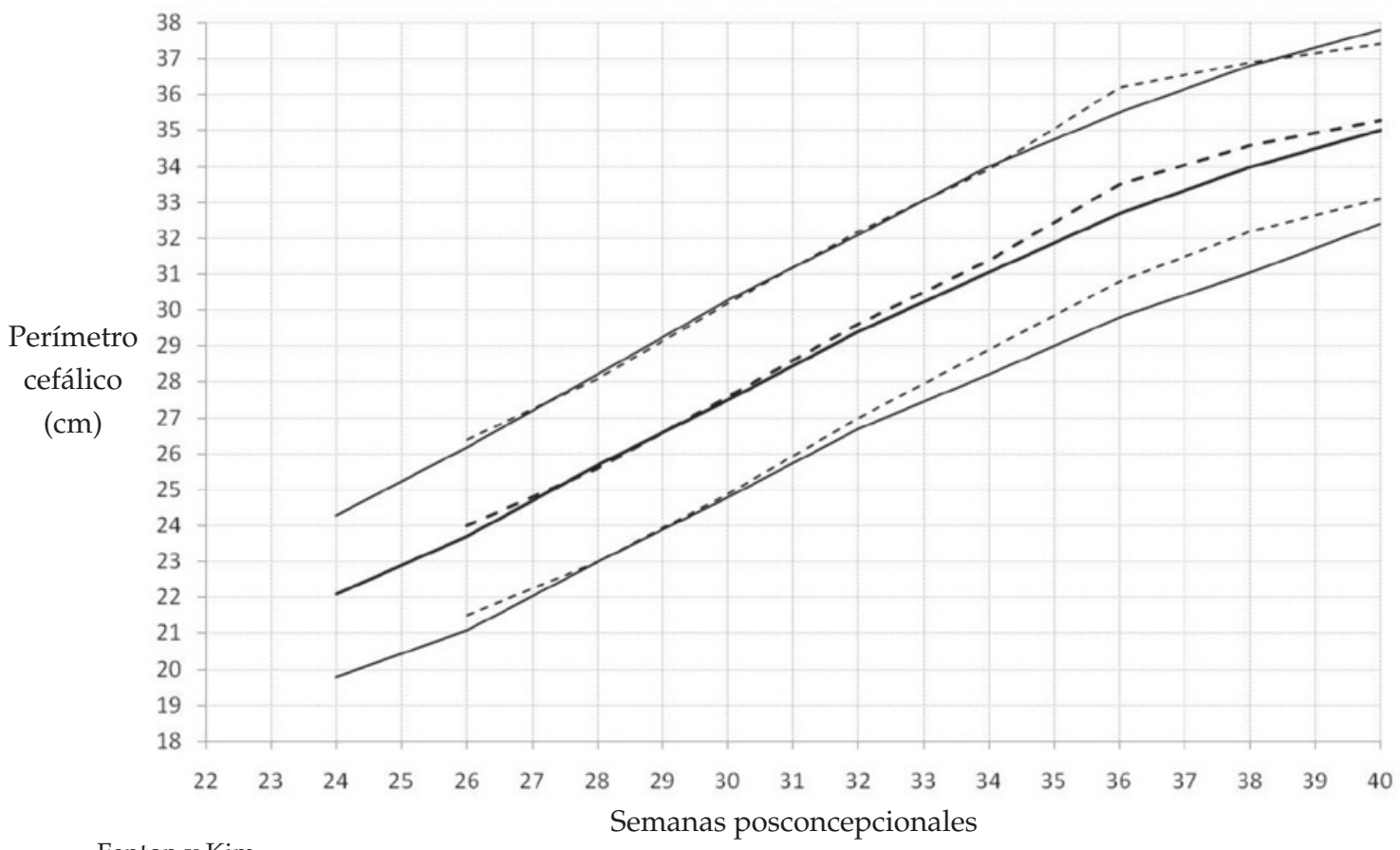


entre las 33 y las 42 semanas de EG.

Mikolajczyk y col., ${ }^{23}$ publicaron, en el año 2011, referencias de peso fetal y peso de RN que pueden ser adaptadas fácilmente a los datos de distintas poblaciones. Para ello, se tiene en cuenta el peso promedio a la semana 40 de EG de cada país, a partir de lo cual se calculan los percentilos para cada EG. Este método permite, además, el ajuste según otros factores, como estatura y peso materno, paridad y sexo del RN.

En las Figuras 1 a 6 , se encuentran graficadas comparativamente las curvas de Lejarraga y Fustiñana ${ }^{4}$ y las de Fenton. ${ }^{21}$ Con respecto al peso, y en ambos sexos, se muestra que el peso de nacimiento promedio es menor en las curvas de Fenton. ${ }^{21}$

Con respecto a la LC, los niños de las curvas de Fenton ${ }^{21}$ tienen menor LC en relación con los datos de Lejarraga y Fustiñana. ${ }^{4}$ En cuanto a PC, los datos de Fenton ${ }^{21}$ son menores que los de Lejarraga y Fustiñana. ${ }^{4}$

\section{CONCLUSIÓN}

Las curvas de Fenton y Kim disponibles entre las 22 y las 50 semanas posconcepcionales tanto para niñas como niños se encuentran disponibles en castellano en http:/ / ucalgary.ca/fenton/files / fenton/espanol-fenton2013growthchart-ninas. pdf y http://ucalgary.ca/fenton/files/fenton/ espanol-fenton2013growthchart-ninos.pdf.

Estas curvas empalman en la semana 50 posconcepcional con las curvas para niños de término en su semana 10 posnatal, curvas adoptadas por la Sociedad Argentina de Pediatría ${ }^{24}$ y el Ministerio de Salud. ${ }^{25}$ Además, en un trabajo reciente de Fustiñana et al. ${ }^{26}$ no se encontraron diferencias clínicas en la aplicación de las curvas de la OMS a niños nacidos prematuros para su seguimiento clínico, lo cual fortalece aún más el criterio de combinar ambas curvas.

El uso de las curvas de Fenton disminuiría el número de niños clasificados como pequeños para la edad gestacional (PEG) en edades tempranas (< de 37 semanas de EG para peso y entre las 34 y las 40 semanas de EG para LC), lo que reduciría la población de niños a la que, a futuro, se podría indicar hormona de crecimiento como tratamiento si no realiza crecimiento compensatorio.

Sobre estas bases es que proponemos y avalamos el reemplazo de las anteriores curvas de Lejarraga y Fustiñana ${ }^{4}$ por las de Fenton y $\mathrm{Kim}^{21}$ para el seguimiento del RN prematuro durante la internación y posalta.

\section{REFERENCIAS}

1. World Health Organization, Expert Committee on Maternal and Child Health. Public health aspects of low birth weight. World Health Organization. Technical Report Series 1961;217:3.

2. Lubchenco L, Hansman C, Dressler M, Boyd E. Intrauterine growth as estimated from liveborn birth weight data at 24 to 42 weeks of gestation. Pediatrics 1963;32:793-800.

3. Lejarraga H, Diaz Ballvé C, Guerra A. Estudio antropométrico de 1401 recién nacidos sanos del área urbana de Buenos Aires. Rev Hosp Niños (B Aires) 1976;15:9-21.

4. Lejarraga H, Fustiñana C. Estándares de peso, longitud corporal y perímetro cefálico desde las 26 semanas hasta las 92 semanas de edad post menstrual. Arch Argent Pediatr 1986;84(4):210-4.

5. Fustiñana C. Evaluación del crecimiento. En Guía de seguimiento del recién nacido de riesgo. Buenos Aires: Ministerio de Salud, 2001:40-51.

6. Comité Nacional de Crecimiento y Desarrollo. Guías para evaluación del crecimiento físico. $3 .{ }^{\text {ra }}$ ed. Buenos Aires: Sociedad Argentina de Pediatría, 2013. [Acceso: 12 de agosto de 2016]. Disponible en: http://www.sap.org.ar/docs/ publicaciones/libro_verde_sap_2013.pdf.

7. Grandi C, López F. Estimación de la edad gestacional: revisión de la literatura. Rev Hosp Mat Inf Ramón Sardá 2004;23(3):138-43.

8. Villar J, Knight HE, De Onis M, Bertino E, et al. Conceptual issues related to the construction of prescriptive standards for the evaluation of postnatal growth of preterm infants. Arch Dis Child 2010;95(12):1034-8.

9. Recomendaciones para el manejo del embarazo y el recién nacido en los límites de la viabilidad. Buenos Aires: Ministerio de Salud, 2007. [Acceso: 12 de agosto de 2016]. Disponibleen:http:/ /www.msal.gov.ar/images/stories/ bes/graficos/0000000513cnt-viabilidad.pdf.

10. Organización Mundial de la Salud. El estado físico: uso e interpretación de la antropometría. Informe de un Comité de Expertos de la OMS. Serie de Informes Técnicos, N. ${ }^{\circ} 854$. Ginebra: Organización Mundial de la Salud; 1995:121-60.

11. Dancis J, O'Connell JR, Holt LE Jr. A grid for recording the weight of premature infants. J Pediatr 1948;33(11):570-2.

12. Casey PH, Kraemer HC, Bernbaum J, Yogman MW, et al. Growth status and growth rates of a varied sample of low birth weight, preterm infants: a longitudinal cohort from birth to three years of age. J Pediatr 1991;119(4):599-605.

13. Wright K, Dawson JP, Fallis D, Vogt E, et al. New postnatal growth grids for very low birth weight infants. Pediatrics 1993;91(5):922-6.

14. Ehrenkranz RA, Younes N, Lemons JA, Fanaroff JA, et al. Longitudinal growth of hospitalized very low birth weight infants. Pediatrics 1999;104(2 Pt 1):280-9.

15. Sauer PJ. Can extrauterine growth approximate intrauterine growth? Should it? Am J Clin Nutr 2007;85(2):608S-13S.

16. Zaw W, Gagnon R, da Silva O. The risks of adverse neonatal outcome among preterm small for gestational age infants according to neonatal versus fetal growth standards. Pediatrics 2003;111(6 Pt 1):1273-7.

17. Cooke RW. Conventional birth weight standards obscure fetal growth restriction in preterm infants. Arch Dis Child Fetal Neonatal Ed 2007;92(3):F189-92.

18. Bertino E, Milani S, Fabris C, De Curtis M. Neonatal anthropometric charts: what they are, what they are not. Arch Dis Child Fetal Neonatal Ed 2007;92(1):F7-10.

19. San Pedro M, Grandi C, Larguía M, Solana C. Estándar de peso para la edad gestacional en 55706 recién nacidos sanos de una maternidad pública de Buenos Aires. Medicina (B Aires) 2001;61:15-22. 
20. Urquía ML, Alazraqui M, Spinelli HG, Frank JW. Referencias poblacionales argentinas de peso al nacer según multiplicidad del parto, sexo y edad gestacional. Rev Panam Salud Pública 2011;29(2):108-19.

21. Fenton TR, Kim JH. A systematic review and meta-analysis to revise the Fenton growth chart for preterm infants. BMC Pediatr 2013;13:59.

22. Villar J, Cheikh Ismail L, Victora CG, Ohuma EO, et al. International standards for newborn weight, length, and head circumference by gestational age and sex: the Newborn Cross-Sectional Study of the INTERGROWTH-21 ${ }^{\text {st }}$ Project. Lancet 2014;384(9946):857-68.

23. Mikolajczyk RT, Zhang J, Betran AP, Souza JP, et al. A global reference for fetal - weight and birthweight percentiles. Lancet 2011;377(9780):1855-61.
24. ComitéNacional de Crecimiento y Desarrollo. La Sociedad Argentina de Pediatría actualiza las curvas de crecimiento de niñas y niños menores de 5 años. Arch Argent Pediatr 2008;106(5):462-7.

25. Argentina. Ministerio de Salud. Resolución 1376/2007. Adopción de los patrones de crecimiento dela Organización Mundial de la Salud para niños. [Acceso: 12 de agosto de 2016]. Disponible en: http://test.e-legis-ar.msal.gov.ar/ leisref/public/showAct.php?id=7105\&word=\#'.

26. Fustiñana C, Rodríguez D, Mariani G. Evaluación pos alta del crecimiento en prematuros. Implicaciones de adoptar las curvas OMS. Arch Argent Pediatr 2014;112(2):141-6

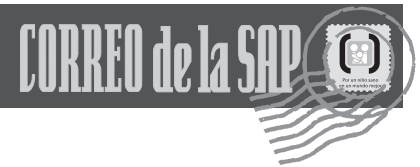

Los siguientes resúmenes y comentarios de trabajos seleccionados se encuentran disponibles en la versión electrónica de este número.

PEDIATRICS 2016;138(3):e20161309

Infecciones por rhinovirus en los 2 primeros años de vida (Toivonen $L$, et al. Rhinovirus infections in the first 2 years of life)

Comentario: Dr. Santiago M. Vidaurreta. Departamento de Pediatría. Hospital Universitario CEMIC. CABA.

ARCH DIS CHILD 2016;101(8):694-700

¿Impacta el uso de la oximetría de pulso en los resultados en salud? Una revisión sistemática

(Enoch AJ, et al. Does pulse oximeter use impact health outcomes? A systematic review)

Comentario: Dra. Hilda Giugno. Servicio de Neumonología. Hospital de Pediatría Juan P. Garrahan. CABA.

N ENGL J MED 2016;375:411-21

Inmunidad innata y riesgo de asma en niños de granjas Amish y Hutterites (Stein MM, et al. Innate immunity and asthma risk in Amish and Hutterite farm children)

Comentario: Dr. Ricardo J. Saranz. Servicio de Alergia e Inmunología, Clínica Universitaria Reina Fabiola. Córdoba.

N ENGL J MED 375;7 August 18, 2016

Paracetamol versus Ibuprofeno en niños menores de cinco años con asma persistente moderada

(Sheehan WT, et al. Acetaminophen versus Ibuprofen in young children with mild persistent asthma)

Comentario: Dr. Alberto Maffey. Centro Respiratorio, Hospital de Niños Dr. Ricardo Gutiérrez. CABA.

N ENGL J MED 2016 Sep 22;375(12):1142-51

Tratamiento con alto flujo nasal para el soporte respiratorio inicial en niños prematuros (Roberts CT, et al. Nasal high-flow therapy for primary respiratory support in preterm infants)

Comentario: Dr. Agustin Bernatzky y Dr. Gonzalo Mariani. Servicio de Neonatología, Hospital Italiano de Buenos Aires. CABA.

N ENGL J MED 2016;375:840-9

Seguridad del salmeterol mas propionato de fluticasona en niños con asma (Stempel DA, et al. Safety of adding salmeterol to fluticasone propionate in children with asthma)

Comentario: Dr. Alejandro Teper y Dr. Daniel Chang. Centro Respiratorio "Dr. Alberto Álvarez".

Hospital de Niños Dr. Ricardo Gutiérrez. CABA. 